\title{
Provisioning Quality of Service for Multimedia Applications in Cloud Computing
}

\author{
Muhammad Usman Ashraf \\ Department of Computer Science, King Abdul Aziz University, Jeddah, Saudi Arabia. \\ E-mail: m.usmanashraf@yahoo.com \\ Sabah Arif \\ Department of Computer Science, Superior University Lahore, Lahore, Pakistan \\ E-mail: sabah.arif@superior.edu.pk

\begin{abstract}
Abdul Basit
Department of Computer Science, The University of Sargodha, Gujranwala, Pakistan. E-mail: pakbasit2000@gmail.com
\end{abstract} \\ Malik Sheraaz Khan \\ Department of Computer Science, The University of Lahore, Lahore, Pakistan. \\ E-mail: Maliksheeraz01@gmail.com
}

Received: 20 March 2017; Accepted: 19 March 2018; Published: 08 May 2018

\begin{abstract}
Since the last decade, many new trends have been introduced to access network technologies and services through internet. Cloud computing is one of those significant technologies that reduce the cost and increase the productivity by providing a variety of services. Recently, cloud computing based system is primarily used for multimedia applications. Over the cloud computing, multimedia applications has some significant quality of service (QoS) requirements such as bandwidth, jitter, latency etc. But due to some limitations in services providing, it is constantly complex to make selection for an appropriate service. Keeping in view the provision of multimedia services through cloud computing, many different concepts and approaches that provide better cloud services under the constraints of QoS attributes have been described in the literature. The goal of this paper is to assess the applicability and provision of multimedia applications over cloud computing through enhanced quality of service. We have identified the primary quality of service msetrics evaluation of multimedia services over cloud computing. Furthermore, under these metrics we evaluated the existing approaches that provide multimedia related services with their strengths and limitations. This evaluation approach could provide the service that can provide better QoS in multimedia applications over cloud computing.
\end{abstract}

Index Terms-Cloud computing, Quality of Services (QoS), Multimedia, bandwidth, jitter, latency, network service, Service level agreement, virtualization, PaaS, SaaS, IaaS.

\section{INTRODUCTION}

Cloud computing is one of most interactive trend in IT that provide the opportunely to run the applications and cloud services on distributed network using virtualized resources and to access these services we use common internet protocols and network standards. In cloud computing, users can access the services and resources which are resided across the internet and you have no need to worry about the maintenance of physical resources So, under the cloud computing paradigm, we can access hardware's, computing resources, and development environment and user applications remotely over the internet and pay for usage only. The services are added quickly on demand and release the resources when not in use [2].

The Cloud computing paradigm is very useful for multimedia applications over the internet such as video conferencing, surveillance where video streaming is required. In order to meet the requirements of clients and services, it is necessary to provide a certain level of QoS by the service provider Such services are device independent where multimedia data is streamed through network and saved cloudily which is accessible from anywhere in the future over the network. According to traditional way, the multimedia data is stored on dedicated servers which are designed for peak usage. Hence a major obstacle for cloud computing system to provide better quality of service is to develop the novel layers that could have ability to interact among cloud service provider and the user. By multimedia over cloud system perspectives, the fundamental aspect is how to achieve better QoS which could be supportive for multimedia applications 
and services over the network [10]. Moreover by future perspectives, many new High Performance Computing approaches are being introduced to overcome multimedia application challenges over cloud computing [34]. Based on HPC many approaches were proposed that can deal such cloud based applications [35, 36].

In order to provisioning such QoS, a lot of companies are working on cloud computing technology to fulfil the user demand under then QoS parameters constraints such as: Netflix, YouTube, Rulu, and Facebook etc. In order to provision of QoS for multimedia services in cloud computing, a variety of models and architectures are proposed. We have made a widespread survey on QoS for multimedia applications in Cloud computing with respect to their proposed frameworks, strengths and Limitations. We also described the cloud computing architecture that is the base architecture in order to provision of QoS for multimedia applications.

Further, other sections of this paper are organized in such a way that, section II describe the basic architecture for cloud computing. Section III explains the others contribution in provision QoS for multimedia applications in cloud computing. In section IV, we have presented a comprehensive analysis of proposed solutions with their strong points and weakness and then conclusion and future directions in last $\mathrm{V}$ section is presented.

\section{BASIC ARCHITECTURE OF CLOUD COMPUTING}

Basically, cloud computing comprises of three fundamental layered architectures including Infrastructure as a Service (IaaS) which is considered as the bottom layer in cloud computing system, Platform as a Service (PaaS) the middle layer in architecture and the third top most layer Software as a Service (SaaS) [1].

In current trend, the technologies that involve the provisions of QoS for multimedia applications are majorly following the cloud computing architecture as a base architecture [1]. Therefore a brief description of this base architecture is as follows:

\section{A. IAAS}

The bottom layer IaaS (Infrastructure as a Service) in cloud computing architecture is creditworthy to provide the physical infrastructure (such as VMs, Hardware resources, storage, network resources) by a vendor which you can access over internet and use to install your software, build or deploy your applications i.e.: Amazon Rackspace. The suitable situations for IaaS to be applicable are as follows:

- When demand is volatile - that is, any time there are significant spikes and troughs of demand on the infrastructure

- For new organizations without the capital to invest in hardware

- When the organization is growing rapidly and scaling hardware would be problematic
- When there is pressure on the organization to limit capital expenditure and to move to operating expenditure

- For specific line of business, trial, or temporary infrastructure needs

Furthermore there are several characteristics for IaaS layer in a cloud computing system which are given as below:

- Resources distributed as a service

- Allows for dynamic scaling

- Has a variable cost, utility pricing model

- Generally includes multiple users on a single piece of hardware

\section{B. PAAS}

The second and middle layer PAAS (Platform as a service) that is essential part of cloud computing architecture which is capable to deliver the deployment service for user application over the cloudy system. PaaS provides a server along with software environment such as development tools, video on demand, databases etc. The most famous PaaS service provider such as Microsoft Azure platform [32] which is facilitate the users to deploy the applications. Another one as Google App engine [33] contained built in services to build a scalable application. PaaS layer could be idealistic in following circumstances:

- The application needs to be highly portable in terms of where it is hosted.

- Proprietary languages or approaches would impact the development process.

- A proprietary language would hinder later moves to another provider (concerns about vendor lockin).

- Application performance requires customization of the underlying hardware and software

\section{SAAS}

The third and most upper layer SaaS (Software as a Service) is responsible to provide the services such as word processing. During interaction between service provider and the user, set of Service level agreements (SLAs) are used to determine that at what level of performance and QoS is demanded by the user. Some fundamental characteristics of SaaS layer are as follows:

- Web access to commercial software

- Software managed from a central location

- Software delivered in a "one to many" model

- Users not required to handle software upgrades and patches

- Application Programming Interfaces (APIs) allow for integration between different pieces of software. 


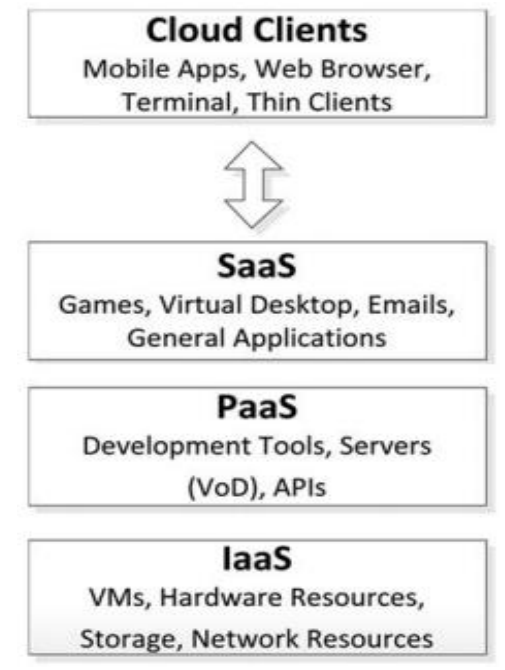

Fig.1. Cloud Service Layered Architecture [2]

Figure 1, clearly shows how users interact with cloud computing paradigm and use the services as required. Moreover, it describes that how all three fundamental layers are organized to provide different services over a cloud. These layers are considered and being follow for any large scaled cloud system. Based on these layers and leading to QoS in cloud, we have defined different QoS metrics for multimedia applications which have been described in next section.

\section{QOS Metrics FOR ClOUdy Multimedia EVALUATION}

Distinctly the selection of an appropriate framework depend on provision of its features that could be evaluated through fundamental metrics [30]. In this section, we have presented the fundamental aspects and their perspective QoS metrics through that the services (which provide QoS for multimedia applications over cloud computing) could be evaluated. Ordinarily, QoS in multimedia applications is evaluated on the base of some limited functional and non-functional quality attributes. Our taxonomy of QoS metrics based on four fundamental aspects including service performance [16][17][18][19] presented in table 1, economics [20][21][22] described in table 2, security [24][25][26] described in table 3 and , QoS attributes [27][28] (such as bandwidth, jitter, latency) presented in table 4 as follows below:

Symbols description: ' $\mathbf{x}$ ' states that the corresponding parameter is in failure while ' $\checkmark$ ' state that attribute working fine.

Table 1. Performance Based Metrics

\begin{tabular}{cccl}
\hline \multicolumn{3}{l}{ QoS Metrics (performance) } & \\
\hline Comp.Time & Stor.Rsp & C.Time & \\
\hline$\checkmark$ & $\checkmark$ & $\checkmark$ & Description \\
$\checkmark$ & $\checkmark$ & $\times$ & Communice performance in Data streaming, processing and storage response is perfect. \\
$\checkmark$ & $\times$ & $\checkmark$ & Streamed data problem could cause of over streaming and delay in packets. \\
$\checkmark$ & $\times$ & $\times$ & Service not responding properly due to problem during streaming and local access. \\
$\times$ & $\checkmark$ & $\checkmark$ & Service performance decreased due to low computation but no packet loss. \\
$\times$ & $\checkmark$ & $\times$ & Performance is almost zero due to neither computation and nor streaming properly. \\
$\times$ & $\times$ & $\checkmark$ & Performance affected by delay in throughput. \\
$\times$ & $\times$ & $\times$ & Service doesn't perform. \\
\end{tabular}

Table 2. Economics Based Metrics

\begin{tabular}{|c|c|c|c|}
\hline \multicolumn{3}{|c|}{ QoS Metrics (Economics) } & \multirow{2}{*}{ Description } \\
\hline T.Cost & C.I.Ratio & Thr.Cost & \\
\hline$\checkmark$ & $\sqrt{ }$ & $\checkmark$ & Economically, service performing in senses. \\
\hline$\checkmark$ & $\checkmark$ & $x$ & In average, service do perform well but not individually. \\
\hline$\checkmark$ & $x$ & $\checkmark$ & Service perform within budget but ratio wise cost increased. \\
\hline$\checkmark$ & $x$ & $x$ & In average, service is inexpensive but not tolerable by throughput. \\
\hline$x$ & $\checkmark$ & $\checkmark$ & Service is efficient as per ratio and individually but over cost exceeds. \\
\hline$x$ & $\checkmark$ & $x$ & Rate of service input/output is efficient but unit wise and overall service expensive. \\
\hline$x$ & $x$ & $\checkmark$ & Overall service is costly but outcome of cost per unit is better. \\
\hline$x$ & $x$ & $x$ & Service couldn't perform within the budget. \\
\hline
\end{tabular}


Table 3. Security Based Metrics

\begin{tabular}{|c|c|c|c|}
\hline \multicolumn{4}{|c|}{ Abbreviations: Total Cost (T.Cost) [23], Cost Incremental Ratio (C.I.Ratio), Throughput Cost (Thr. Cost) } \\
\hline \multicolumn{3}{|c|}{ QoS Metrics (Security) } & \multirow{2}{*}{ Description } \\
\hline Conf. & Sens. & Auth. & \\
\hline$\checkmark$ & $\checkmark$ & $\checkmark$ & Service fully secured. \\
\hline$\checkmark$ & $\checkmark$ & $x$ & Verification from destination is not sure in service but data streaming is secured. \\
\hline$\checkmark$ & $x$ & $\checkmark$ & Data stream is entrusted and verified but need to \\
\hline$\checkmark$ & $x$ & $x$ & Provided service is confidential but acquired verification. \\
\hline$x$ & $\checkmark$ & $\checkmark$ & Service provides validation from source/destination but required concealment. \\
\hline$x$ & $\checkmark$ & $x$ & Streaming data is untrusted and non-verified but unsusceptible. \\
\hline$x$ & $x$ & $\checkmark$ & Data is verified but streamed over untrusted and susceptible service. \\
\hline$x$ & $\mathbf{x}$ & $\mathbf{x}$ & Service is fully unsecured. \\
\hline
\end{tabular}

Abbreviations: Confidentiality (Conf.), Sensitivity (Sens.), Authentication (Auth.) [24][25][26][29][31]

\section{QOS PROVISION APPROACHES FOR MULTIMEDIA}

Hongli, Aaron and Timothy.[1] proposed an architecture for private multimedia cloud computing using virtualization infrastructure and addressed variety of challenges including heterogeneity of network and limitation of mobile devices for provisioning QoS for multimedia applications. They implemented the QoS provisioning mechanism in IaaS layer of proposed model. The proposed virtualized video streaming server architecture provides a high performance under limited cost but over a fully no implementation of all QoS parameters when it is utilized across heavy traffic over the network.

P. Manoj et al. [2] continued effort on service delivery framework for cloud service management and resource allocations automatically. By utilizing this proposed framework, it help out to manage extra consumption and reduce jitter for cloud based multimedia services. According to this framework, the basic concept is to run the cloud services on public cloud depending on network status and client's service demand. This scalable framework provides many features including availability on demand, load-balancing and robustness. In this why, a heavy traffic over the network could be prevented. The proposed model accomplishes three major functions of MM-aware cloud as follows:

- Providing and supporting the QoS

- QoS adaptation.

- Process the applications in parallel in distributed system.

Pei-Jia Yang and Yang-Fang.[3] proposed cloud computing based model an architecture "IMS Cloud system architecture" to access high quality multimedia services via android base appliances. They used service prioritizing mechanism and Improve system performance. They focus on heterogeneity and mobility issues using IMS QoS policy and overall video stream throughput increased using proposed architecture. The proposed model was implementable for android platform only.

Fragkiskos et al.[4] introduced a layered architecture system that relates to OSI layers model for cloud resources management in provision better QoS for multimedia applications. The proposed SPM mechanism have multiple features in QoS provisioning. It provides automated resources allocation service which is optimized and consume less bandwidth by reducing jitter. But found a drawback of increasing latency on user movement while streaming a video.

Anand V Akella and Kaiqi Xiong.[5] proposed a SDN (Software defined networking) based approach called "Open VSwitch". According to authors, the proposed model provides an opportunity to automatically switch on available path for higher priority clients to ensure the guarantee of QoS with multiple parameters such as bandwidth, number of hops and RTT. They implemented and tested the proposed model in GENI (Global Environment for Networking Innovations) but not by using Open Flow physical switch.

Michael et al. [6] introduced a new PAAS architecture targeting real-time QoS guarantees for online interactive multimedia applications. There were two major key aspects of proposed PaaS as QoS oriented Service Engineering and on demand service management. But there were some weakness for dynamic uncertainty management. This PaaS architecture is still in progress to improve the quality of service and being verified as well.

K.Saravanan and M.Lakshmi Kantham.[7] proposed an approach for advance reservation and ranking of multimedia cloud services using QoS attributes. They emphasized on QoS provision when a vast number of users request for cloud services. To overcome this issue, they proposed ranking technique. In proposed model, they explained all the QoS characteristics but not used during implementation.

S.Kumar, S.Versteeg and Rajkumar.[8] targeted quality measurement and prioritization of multimedia cloud services and proposed AHP (Analytical Hierarchical Process) based framework. In context of provision SLA and QoS, this framework create a strong competition and impacted. Authors proposed taxonomy of QoS attributes. Hence each attribute might be fall in quantifiable or nonquantifiable category. According to authors, the proposed approach is applicable for only quantifiable attributes but facing several challenges during implementation for nonquantifiable attributes.

Ruozhou et al.[9] proposed different algorithms to 
make an appropriate selection for QoS-aware cloud services. Generally, a cloud computing system contained tailor-made MM cloud services through which a user could interact with cloud system. Cloud computing services are not only limited to provide the services but also interconnection among these cloud services as well. To overcome this issue, they proposed number of algorithms to select the service automatically as requested. Provision of QoS is enhanced using these algorithms and virtualization mechanism but some other major parameters were skipped like latency and jitter.

Wenwu et al [10] addressed the challenges

Worked on cloud services for multimedia over cloud computing and addressed number of challenges in QoS provisioning. In order to improve the better quality of service for multimedia applications in cloud, authors proposed a new architecture as "MEC (Media Edge Cloud)-computing architecture". Moreover, the cloud media resources could be used optimally using the proposed architecture. They also described the some future directions and problems for multimedia cloud computing and how MEC architecture could be enhanced to get better QoS of multimedia applications in cloud computing.

W.Hui, C.Lin and Yang [11] worked on three major challenges for Multimedia cloud computing such as "heterogeneity, scalability, and multimedia QoS provisioning" and proposed a new architecture as "MediaCloud". They also introduced the key technologies through which users could be provided multimedia applications and services effectively and efficiently with QoS provisioning along MediaCloud architecture. MediaCloud was designed particularly to process complex services with efficient resource allocation, scalability, and QoS provisioning. According to authors, there are still number of challenges in order to determining quality of experience (QoE) of multimedia services and how to address the potential security threats.

Yee Chen, Yi Peng.[12] worked on finding optimal service path route and proposed a QoS Aware Services Mashup(QASM) model. According to authors, QoS aware path routing is an NP-Hard problem so it must be explored deeply and fixed. They implemented the proposed model as a simulation test witch's results clarify the correctness of proposed model and algorithm and will implement a prototype of their model in a real internet environment. In actual implementation, it is important to investigate that how to include some other system properties such as stability and fault tolerance.

C. Lai, H. Wang, H. Chao and G. Nan. [13] Proposed a new system architecture for cloud based media streaming to maintain the multimedia QoS for a certain level. They proposed two interactive modules Network and Device-
Aware Bayesian Prediction Module (NDBPM) used for the prediction of network and hardware features and Dynamitic Network Estimation Module (DNEM) to control switch virtual circuit (SVC) multimedia steaming and communication frequency. According to authors, the proposed solution assures for a smooth and complete multimedia streaming services. But SVC coding scheme still required a lot of consideration to make more refinement for cloud media streaming.

I. Trajkovska, J. Salvachúa and A. Velasco, [14] introduced a cloud media API that contains the built in functions to calculate QoS and its parameters automatically among cloud media service providers and its prospective clients. The proposed architecture is basically joiner of peer to peer and cloud computing that support for multimedia streaming in both CS (cloud streaming) and P2P style. Due to less implementations of proposed API, there is an open issue to implement proposed API in a cloud infrastructure by extending Isabel system functionality and also to test its consequence behavior.

A. Li, X. Yang, S. Kandula and M. Zhang [15] discussed on performance monitoring and analysis tools for multimedia application in cloud computing paradigm. These tools can be used to measure the QoS for media cloud services and data ranking as well. Furthermore, these tools also applicable to make performance comparison between different cloud services such as Rackspace, windows azure and Amazon EC2 etc. During comparison they emphasized on resources utilization, network throughput and QoS parameters at a low level performance of cloud services. Conversely, they didn't deploy the more constraints in order to achieve high QoS for multimedia applications in cloud computing.

\section{COMPARATIVE ANALYSIS AND RESUltS}

In this section, we have made a comprehensive analysis of proposed approaches in era of QoS for multimedia in cloud computing. We have described the proposed approach with its strong points and limitations as well. Some of the techniques are very useful and directed in very right way but still required a lot of concentration to in order to get targeted outcome. Most of multimedia frameworks have still weaknesses in many significant parts to achieve a high QoS for multimedia applications over the network. For such a frameworks and models, there are number of open issue for future research and directions. To review the proposed approaches and its perspective strong points and limitations, follow the below: table 5 . 
Table 5. Evaluation of proposed techniques and frameworks

\begin{tabular}{|c|c|c|c|}
\hline Ref. & Proposed Approaches & Strong Points & Limitations \\
\hline 1 & $\begin{array}{l}\text { Architecture of Multimedia cloud } \\
\text { computing }\end{array}$ & All QoS attributes are considered only. & $\begin{array}{l}\text { Not tested at large level. } \\
\text { No implementation in real network }\end{array}$ \\
\hline 2 & Service delivery framework & $\begin{array}{l}\text { QoS support, provisioning and parallel } \\
\text { processing for distributed systems. }\end{array}$ & $\begin{array}{l}\text { No implementation at large network. } \\
\text { Performance and economically expensive. }\end{array}$ \\
\hline 3 & IMS Cloud system architecture & $\begin{array}{l}\text { Video stream throughput increased } \\
\text { Fixed heterogeneity and mobility }\end{array}$ & $\begin{array}{l}\text { Implementable only for specific platform } \\
\text { "android" } \\
\text { Facing Performance challenge. }\end{array}$ \\
\hline 4 & $\begin{array}{l}\text { Layered system architecture relates to } \\
\text { OSI }\end{array}$ & $\begin{array}{l}\text { automated resource allocation reduce } \\
\text { bandwidth and jitter }\end{array}$ & $\begin{array}{l}\text { increase of latency as a user moves while } \\
\text { streaming }\end{array}$ \\
\hline 5 & Open vSwitch based algorithm & $\begin{array}{l}\text { Switch service automatically priority base } \\
\text { including perms (bandwidth, RTT, and the } \\
\text { no. of hop). }\end{array}$ & $\begin{array}{l}\text { Not implemented and tested by using Open } \\
\text { Flow physical switch }\end{array}$ \\
\hline 6 & New PAAS architecture & $\begin{array}{l}\text { QoS oriented Service Engineering } \\
\text { On demand service management }\end{array}$ & $\begin{array}{l}\text { Lake of assurance for dynamic uncertainty } \\
\text { management } \\
\text { Still in progress to improve the quality of PaaS }\end{array}$ \\
\hline 7 & $\begin{array}{l}\text { Ranking and advanced reservation } \\
\text { framework }\end{array}$ & $\begin{array}{l}\text { Explained basic QoS attributes such as } \\
\text { bandwidth, jitter, etc. }\end{array}$ & $\begin{array}{l}\text { No implementation practically for mentioned } \\
\text { QoS attributes. }\end{array}$ \\
\hline 8 & $\begin{array}{l}\text { AHP(Analytical Hierarchical Process) } \\
\text { based framework }\end{array}$ & $\begin{array}{l}\text { Approachable only for quantifiable } \\
\text { attributes }\end{array}$ & $\begin{array}{l}\text { Several challenges faced during achieving non- } \\
\text { quantifiable attributes }\end{array}$ \\
\hline 9 & Proposed different algorithms & Automatic service selection on requested. & $\begin{array}{l}\text { Some major parameters skipped like latency } \\
\text { and jitter. }\end{array}$ \\
\hline 10 & $\begin{array}{l}\text { MEC(Media Edge Cloud)-computing } \\
\text { architecture }\end{array}$ & $\begin{array}{l}\text { High Provision QoS } \\
\text { Optimal resource allocation }\end{array}$ & Mentioned future work and problems in MEC. \\
\hline 11 & MediaCloud architecture & $\begin{array}{l}\text { Resource allocation, scalability, and } \\
\text { multimedia QoS provisioning }\end{array}$ & $\begin{array}{l}\text { determining (QoS) of MM services and to } \\
\text { address potential security threats }\end{array}$ \\
\hline 12 & $\begin{array}{l}\text { QoS Aware Services Mashup (QASM) } \\
\text { model }\end{array}$ & Optimal QoS path route allocation & No real internet environment implementation \\
\hline 13 & NDBPM and DNEM Modules & $\begin{array}{l}\text { Provide smooth multimedia streaming } \\
\text { services }\end{array}$ & $\begin{array}{l}\text { SVC coding scheme is not implementable for } \\
\text { large network traffic. Need refinement. }\end{array}$ \\
\hline 14 & Joined (P-2-P and Cloud computing) API & $\begin{array}{l}\text { Calculate QoS and its parameters } \\
\text { automatically by providing media services }\end{array}$ & $\begin{array}{l}\text { Need implementation for Isabel system } \\
\text { functionality and test behavior }\end{array}$ \\
\hline 15 & Performance monitoring and analysis tool & $\begin{array}{l}\text { QoS measurement and at low level } \\
\text { performance analysis }\end{array}$ & $\begin{array}{l}\text { No constraint to deploy in order to achieve high } \\
\text { QoS }\end{array}$ \\
\hline
\end{tabular}

The above evaluation shows how the proposed services having limitations in order to provide QoS for multimedia applications over cloud computing. Majorly QoS attributes and performance aspects are still required a lot of considering by future perspective because still a massive bandwidth is required to deliver better service which directly affect the overall economics of service provision. However, QoS attributes, communication time, computational time and storage response are the primarily areas that should be considered in priority to improve the services for multimedia applications in cloud computing. This evaluation is not limited to just for these listed proposed frameworks but could be implementable to evaluate in future being proposed approaches.

\section{CONCLUSION}

The Cloud computing is one of the new emerging technology which has made a rapid growth to offer cloud based media services to various consumers. Recently, Cloud computing is being used for multimedia applications extensively. It is constantly complex to make selection for an appropriate service that could provide better quality of service for multimedia applications over cloud computing. Distinctly the selection of an appropriate framework depend on provision of its features that could be evaluated through fundamental metrics. We have presented some fundamental QoS metrics that could evaluate the service by different aspects. These QoS metrics are classified into four major aspects including Service performance, service economy, service attributes and service security. Furthermore, through a critical analysis, we have evaluated the strengths and limitations of the existing approaches that provide services for multimedia applications in cloud computing. The proposed evaluation approach could guarantee it that the selected service could provide better QoS or not. By future perspective, our plan is to propose a framework that could provide better QoS by evaluating through these defined metrics.

\section{REFERENCES}

[1] H. Luo, A. Egbert and T. Stahlhut, "QoS Architecture for Cloud-based Media computing”. IEEE. Dec 2012.

[2] S. Vani, B. Bhosale, G. Shinde, R. Shinde and P. Pawar, "Cloud-Based Multimedia Storage System with QoS", International Journal of Computer Science and Information Technologies. vol. 5(2), pp. 1173-1176, 2014

[3] J. Chen, S. Lin, Y. Larosa, P. Yang and Y. Fang "IMS Cloud Computing Architecture forHigh-Quality Multimedia Applications", IEEE, 2011.

[4] F. Sardis, G. Mapp, L. Jonathan, M. Aiash, and A. Vinel, "On the Investigation of Cloud-based Mobile Media Environments with Service-Populating and QoS-aware Mechanisms".IEEE, March 2011.

[5] A.V Akella and K. Xiong, "Quality of Service (QoS) Guaranteed Network Resource Allocation via Software Defined Networking (SDN)", International Conference on 
Dependable, Autonomic and Secure Computing. IEEE,2014.

[6] M. Boniface,"Platform-as-a-Service Architecture for Real-time Quality of Service Management in Clouds," Fifth International Conference on Internet and Web Applications and Services. IEEE. 2010.

[7] K. Saravanan and M.L. Kantham, "An enhanced QoS Architecture based Framework for Ranking of Cloud Services", International Journal of Engineering Trends and Technology. Vol 4 (4). April 2013.

[8] S.K. Garg, S. Versteeg and R. Buyya, "SMICloud: A Framework for Comparing and Ranking Cloud Services," Fourth IEEE International Conference on Utility and Cloud Computing. IEEE. 2011.

[9] Y. Ruozhou.Yang, J. Huang, Q. Duan and Y. Tanaka "QoS-aware Service Selection in Virtualization-based Cloud Computing," International Scientific and Technological Cooperation Program. IEEE. 2013.

[10] Z. Wenwu, L. Chong, J. Wang and L. Shipeng, "An emerging technology for providing multimedia services and applications," Digital Object Identifier. April 2011.

[11] W. Hui, C. Lin and Y. Yang "A New Paradigm of Multimedia Computing," ksii transactions on internet and information systems. Vol 6(4). April 2012.

[12] Y. M. Chen, Y. J. Peng, "A QoS aware services mashup model for cloud computing applications," vol 5(2). Pp. 457-472. 2013.

[13] C. Feng, H. Wang and H Chao, "A Network and Device Aware QoS Approach for Cloud-Based Mobile Streaming, " Transactions On Multimedia.IEEE. Vol 15(4). June 2013.

[14] I. Rajkovska, J. Salvachúa and A.M. Velasco, "A Novel P2P and Cloud Computing Hybrid Architecture for Multimedia Streaming with QoS Cost Functions, ". IEEE. 2012.

[15] A. Li, X. Yang, S. Kandula and M. Zhang, "CloudCmp: comparing public Cloud providers", Published in the Proceedings of the 10th Annual Conference on Internet Measurement, Melbourne, Australia, 2010.

[16] Q. He, S. Zhou, B. Kobler, D. Duffy, and T. McGlynn, "Case study for running HPC applications in public clouds," Proc. 19th ACM Int. Symp. High Performance Distributed Computing (HPDC 2010), ACM Press,Jun. 2010, pp.395-401.

[17] Z. Li, L. O'Brien, R. Cai, and H. Zhang, "Towards a taxonomy of performance evaluation of commercial Cloud services," Proc. 5th Int. Conf. Cloud Computing (IEEE CLOUD 2012), IEEE Computer Society, Jun. 2012, to appear.

[18] M. R. Palankar, A. Iamnitchi, M. Ripeanu, and S. Garfinkel, "Amazon S3 for science grids: a viable solution?," Proc. 2008 Int Workshop on Data-Aware Distributed Computing (DADC 2008), ACM Press, Jun. 2008, pp. 55-64.

[19] S. Ostermann, A. Iosup, N. Yigitbasi, R. Prodan, T. Fahringer, and D. H. J. Epema, "A performance analysis of EC2 Cloud computing services for scientific computing," Proc. 1st Int. Conf. Cloud Computing (CloudComp 2009), Springer-Verlag, Oct. 2009, pp. 115131.

[20] M. Armbrust, A. Fox, R. Griffith, A. D. Joseph, R. Katz, A. Konwinski, G. Lee, D. Patterson, A. Rabkin, I. Stoica, and M. Zaharia, "A view of Cloud computing," Commun. ACM, vol. 53, no. 4, Apr. 2010, pp. 50-58.

[21] T. A. Hjeltnes and B. Hansson, Cost Effectiveness and Cost Efficiency in E-learning, Trondheim, Norway: The TISIP Foundation, 2005.
[22] A. Li, X. Yang, S. Kandula, and M. Zhang, "CloudCmp: Comparing public Cloud providers," Proc. 10th Annu. Conf. Internet Measurement (IMC 2010), ACM Press, Nov. 2010, pp. 1-14.

[23] N. Yigitbasi, A. Iosup, D. Epema, and S. Ostermann, "CMeter: A framework for performance analysis of computing Clouds," Proc. 9th IEEE/ACM Int. Symp. Cluster Computing and the Grid (CCGRID 2009), IEEE Computer Society, May 2009, pp. 472-477.

[24] Q. Zhang, L. Cheng, and R. Boutaba, "Cloud computing: State-ofthe-art and research challenges," J. Internet Serv. Appl., vol. 1, no. 1, May 2010, pp. 7-18.

[25] M. Armbrust, A. Fox, R. Griffith, A. D. Joseph, R. Katz, A. Konwinski, G. Lee, D. Patterson, A. Rabkin, I. Stoica, and M. Zaharia, "A view of Cloud computing," Commun. ACM, vol. 53, no. 4, Apr. 2010, pp. 50-58.

[26] C. Brooks, "Cloud computing benchmarks on the rise," SearchCloudComputing, TechTarget.com, Jun. 10, 2010. [online].

Available:http://searchcloudcomputing.techtarget.com/ne ws/1514547/ Cloudcomputing-benchmarks-on-the-rise.

[27] Zhu, Wenwu, et al. "Multimedia cloud computing." IEEE Signal Processing Magazine 28.3 (2011): 59-69.

[28] Mell, Peter, and Tim Grance. "The NIST definition of cloud computing." (2011).

[29] K. Khan, A.Sahai. A Glowworm Optimization Method for the Design of Web Services, International Journal of Intelligent Systems and Applications (IJISA), 4 (10), 2012, pp. 89-102.

[30] Z. Li, L. O'Brien, R. Cai, and H. Zhang. - Towards a Taxonomy of Performance Evaluation of Commercial Cloud Services, Proceeding 5th Int. Conf. CloudComputing (IEEE CLOUD 2012), IEEE Computer Society, 2012.

[31] Zh. Li, L. O'Brien, H. Zhang and R. Cai. On a Catalogue of Metrics for Evaluating Commercial Cloud Services, 13th International Conference on Grid Computing, ACM/IEEE, 2012, pp. 164- 173.

[32] Armbrust, Michael, et al. "A view of cloud computing." Communications of the ACM 53.4 (2010): 50-58.

[33] Zhang, Qi, Lu Cheng, and Raouf Boutaba. "Cloud computing: state-of-the-art and research challenges." Journal of internet services and applications 1.1 (2010): 7 18.

[34] Ashraf, Muhammad Usman, Fadi Fouz, and Fathy Alboraei Eassa. "Empirical Analysis of HPC Using Different Programming Models." (2016).

[35] Ashraf, Muhammad Usman, and Fathy Elbouraey Eassa. "OpenGL Based Testing Tool Architecture for Exascale Computing." International Journal of Computer Science and Security (IJCSS) 9.5: 238.

[36] Ashraf, Muhammad Usman, and Fathy Elbouraey Eassa. "Hybrid Model Based Testing Tool Architecture for Exascale Computing System."International Journal of Computer Science and Security (IJCSS) 9.5 (2015): 245.

\section{Authors' Profiles}

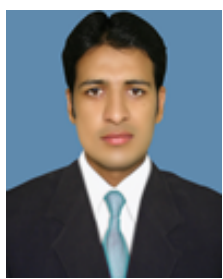

Muhammad Usman Ashraf received his B.Sc degree from Govt. College Gujranwala in 2007, M.Sc degree in Computer Science from The University of Agriculture Faisalabad in 2009 and Master of Science in Computer Science from University of Lahore, Pakistan in 2014. 
Currently, he is doing Ph.D. In computer science from King Abdul-Aziz University Jeddah, Saudi Arabia. His research interests include Exascale computing System, High Performance Computing, Ubiquitous Computing and Context awareness. He has presented many papers in National and International conferences.

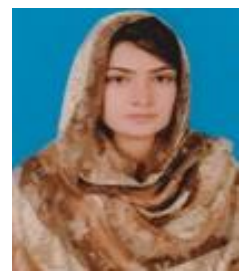

Sabah Arif received her B.S (Hon) degree in Computer Science from The Islamia University of Bhawalpure, Pakistan in 2012 She received her Master degree in Computer Science from The University of Lahore, Pakistan in 2015. She is a permanent Lecturer in the Department of Computer Science, Superior University Lahore, Pakistan. Her research interest includes Software engineering, High Performance Computing, Parallel Computing and Cloud Computing.

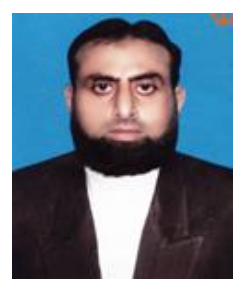

Abdul Basit received his B.Sc degree in Mathematics from the University of Punjab, Pakistan in 1993. He received his Master degree in Computer Science from University of Lahore, Pakistan in 2003. He is a Lecturer in the Department of Computer Science, University of Sargodha (Gujranwala Campus), Pakistan. His research interest includes Computer Networks, Wireless networks, Mobile computing, cloud computing and Big data.

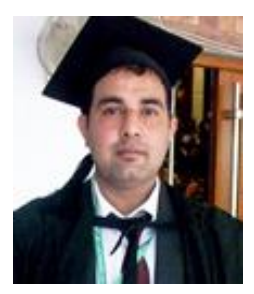

Malik Sheraaz Khan received his BSCS degree from NIIT Islamabad, Pakistan in 2008, MSCS degree in Computer Science from The University of Lahore, Pakistan in 2014. Currently, he is working in NESCOM Islamabad, Pakistan as an IT officer. His research interests include High Performance Computing, Context awareness, Cloud computing and Wireless Networks. He has presented many papers in National and International conferences.

How to cite this paper: Muhammad Usman Ashraf, Sabah Arif, Abdul Basit, Malik Sheraaz Khan, "Provisioning Quality of Service for Multimedia Applications in Cloud Computing", International Journal of Information Technology and Computer Science(IJITCS), Vol.10, No.5, pp.40-47, 2018. DOI: 10.5815/ijitcs.2018.05.04 